\title{
Research on Machinery Fault Diagnostics Based on Vibration Signal Analysis
}

\author{
Lv Yan $^{1, *}$, Fang Liqing ${ }^{1}$ and Zhang Qiantu ${ }^{1}$ \\ ${ }^{1}$ Department of Guns Engineering, Ordnance Engineering College, 050003 Shijiazhuang, China
}

\begin{abstract}
Aiming at the non-stationary features of mechanical vibration signals, a fault diagnosis method based on ensemble empirical mode decomposition singular value entropy and support vector machine are put forward. This method utilizes the advantage of EEMD which can effectively restrain model mixing and combined with information entropy theory, firstly, original signals were decomposed into a finite number of stationary intrinsic mode functions (IFMs). Secondly, singular value and singular entropies of a feature pattern matrix whose rows are IMFs are extracted. Finally, singular value feature vector are served as the input vectors of SVM, based on the output to identify the fault pattern. Experimental results show that this method is affective.
\end{abstract}

\section{Introduction}

The vibration signal of rolling bearing is non-linear, how to extract feature from non-linear is the key point for fault diagnosis[1]. Empirical mode decomposition[2] (EMD) is of great use to analysis non-linear vibration signal, but an open problem of it is the model mixing problem. In 2009, $\mathrm{Wu}$ and Huang proposed an improved version of EMD, called ensemble empirical mode decomposition[3] (EEMD). By adding finite white noise to investigated signal, the EEMD method can eliminate the model mixing. When some occurs in the bearing, the singular entropy will change due to the change of singular value of IMFs, so the singular entropy can be used to specify whether the bearing has fault or not, and the singular value of IMFs can be used as the feature vector.

On the other hand, support vector machine (SVM) is a powerful machine method based on statistical learning theory and structural risk minimization principle, which can overcome the disadvantage of artificial neural network, are widely used in the fault diagnosis of rolling bearing[4-5]. In this paper, a fault diagnosis method based on ensemble empirical mode decomposition singular entropy(EEMD-SE) and support vector machine are put forward. The IMFs which contain almost information of the fault are chosen to initial feature vector matrix, then the singular value vector which is obtained by singular value decomposition (SVD) form the matrix is input to the SVM to specify the fault type.

\section{Ensemble empirical mode decompose- tion (EEMD)}

Let the original signal is $x(t)$, the EMD method is to decompose $x(t)$ into several IMFs $C_{i}$ and a residue $r_{n}$.

$$
x(t)=\sum_{i=1}^{n} c_{i}(t)+r_{n}(t)
$$

The principle of EEMD is sample: the added white noise populates the white time-frequency space uniformly, facilitating a natural of the frequency scales, which reduces the occurrence of mode mixing[6]. The EEMD algorithm is as follow [7].
(1) Add a numerically generated white noise to the original signal to generate a new signal:

$$
x_{k}(t)=x(t)+n_{k}(t)
$$

where $k=1,2, \cdots, N$, and $N$ is the number of ensemble.

(2) Use the original EMD algorithm to decompose the newly generated signal into IMFs.

$$
x_{k}(t)=\sum_{j=1}^{n} c_{k j}(t)+r_{k}(t)
$$

where $n$ is the number of IMFs, $c_{k j}(t)$ represents the IMFs and $r_{k}(t)$ is the final residue.

(3) Repeat steps 1 and 2 with different white noise series each time.

(4) Calculate the ensemble means of the corresponding IMFs of the decomposition as the final result:

$$
\begin{gathered}
c_{j}(t)=\frac{1}{N} \sum_{k=1}^{N} c_{k j}(t) \\
r(t)=\frac{1}{N} \sum_{k=1}^{N} r_{k}(t)
\end{gathered}
$$

The original vibration signal of bearing with outer race fault are shown in Figure 1 and the decomposed signal using EEMD are shown in Figure 2. From Figure 2 we can find that the EEMD method decompose the original signal into 11 IMFs and a residue, each IMFs has different frequency components.

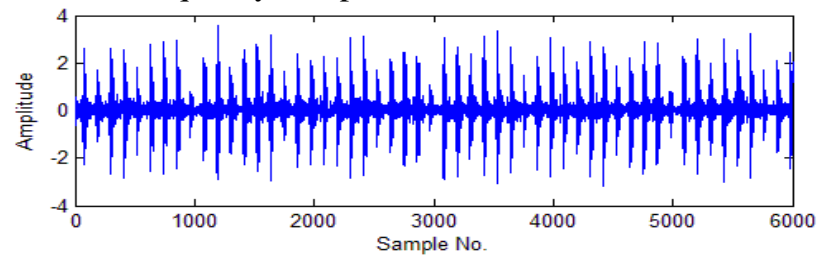

Figure 1. The original vibration signal of bearing with outer race fault 


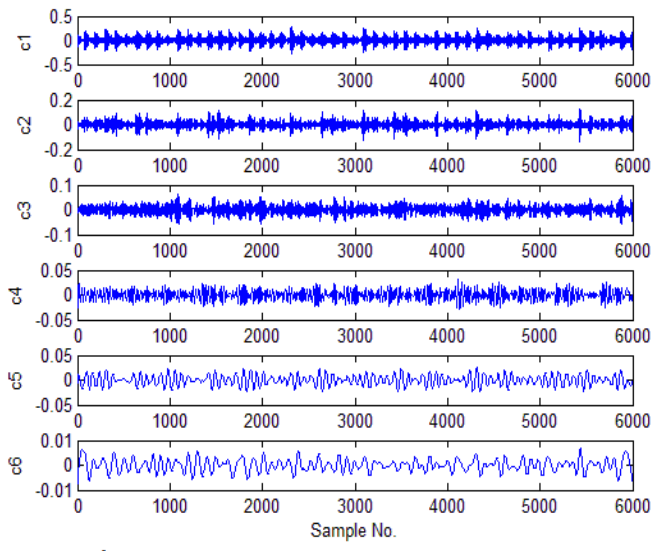

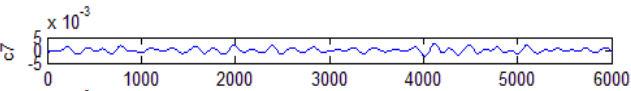
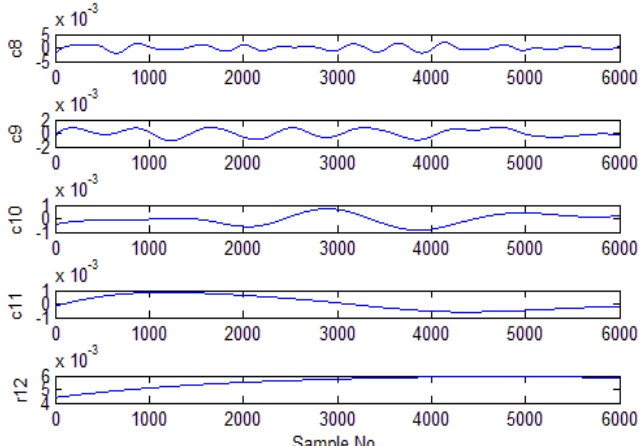

Figure 2. The EEMD decomposed results

\section{Ensemble empirical mode decomposi- tion singular entropy}

Singular value is the nature characteristics of the matrix and own favorable stability, and has the characteristics of scale invariance and rotating invariance. When the different fault occurs in the bearing, the singular value of IMFs decomposed from different fault signal will change. It is very feasible to be the fault feature. Hence, the EEMD singular entropy can be used to specify whether the bearing has fault or not.

Use the EEMD method to decompose the vibration signal $x(t)$ into several IMFs and a residue, each IMF has different frequency components, use them to initial feature vector matrix $M$, and then the singular value $\left(\sigma_{1}^{2} \sigma_{2}^{2} \cdots \sigma_{\mathrm{n}}^{2}\right.$ ) of $M$ are extracted. Combined with information entropy theory, the formula of singular value entropy are given[8]:

$$
\left\{\begin{array}{l}
P=-\sum_{i=1}^{n} p_{i} \log p_{i} \\
p_{i}=\frac{\sigma_{i}^{2}}{p} \\
p=\sum_{i=1}^{n} \sigma_{i}^{2}
\end{array}\right.
$$

The EMD singular entropy and EEMD singular entropy of bearing with different fault are shown in Table
1. We can find that the EEMD singular entropy is smaller than EMD, it is because that EEMD overcome the model mixing problem, the uncertainly of energy distribution are reduced. The entropy of normal bearing is bigger than that bearing with fault, it is because the energy of the normal bearing vibration signal is evenly distributed, so the entropy is bigger. On the contrary, in the fault condition, the energy distribution becomes uneven or certain, so the entropy is smaller. The singular entropy of different fault pattern is different, so we can use it to specify the fault type. But specify the fault type only based on the singular entropy value is not enough, further analysis are needed.

Table1. The EMD-SE and EEMD-SE of rolling bearing in different condition

\begin{tabular}{|c|c|c|c|c|}
\hline Fault type & Normal & Inner race & Ball & Outer race \\
\hline EMD-SE & 1.9969 & 1.6081 & 1.5429 & 1.2264 \\
\hline EEMD-SE & 1.9804 & 1.4363 & 1.3845 & 1.0297 \\
\hline
\end{tabular}

\section{The theory of SVM}

SVM are often used for classification and regression analysis. As for the class problem, the most important is to find the optimal separating plane. A two-class problem are shown in Figure 3, from the Figure 3, we can find that the parallel line between 11 and 12 can correctly separate two samples, they can be the candidate separating line. It is obvious that the line in the middle of 11 and 12 is the best. The best separating line is not only can correctly separate two samples, but also can maximize the margin between separating line. Let the linear equation of line 1 is:

$$
\omega \cdot x+b=0
$$

the margin between 1 and $11(12)$ is $1 /\|\omega\|$, maximize the margin is equal to minimize $\|\omega\|$, then the problem may be handled by solving the following quadratic optimization problem[9]

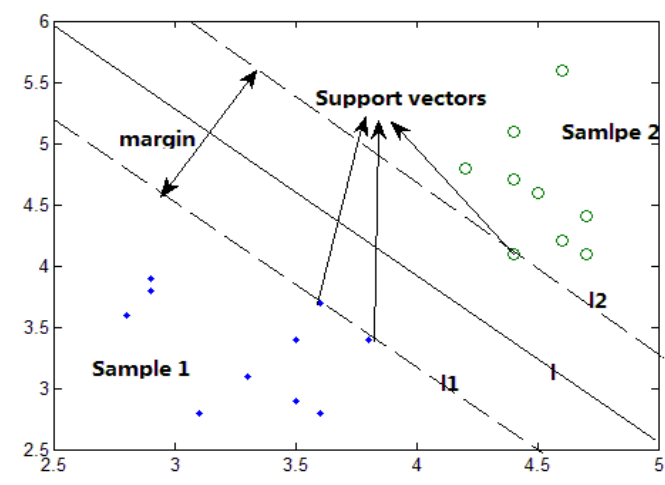

Figure 3. Optimal separating line 


$$
\left\{\begin{array}{c}
\min _{\omega, b} \frac{1}{2}\|\omega\|^{2} \\
\text { s.t. } y_{i}\left(\left(\omega \cdot x_{i}\right)+b\right) \geq 1, i=1, \cdots, n
\end{array}\right.
$$

From Eq (8), the best $\omega^{*}$ and $b^{*}$ can be extracted, then the final decision function can be given by

$$
f(x)=\operatorname{sgn}\left(\left(\omega^{*} \cdot x\right)+b^{*}\right)
$$

\section{Procedures of the proposed method and experimental results}

\section{1 Procedures of the proposed method}

The singular values of the matrix whose rows are IMFs decomposed by EEMD, are input to SVM to classify the fault type. The complete process of the proposed method is given in the following steps. It also is shown in Figure 4.

(1) Collect vibration signals under various condition.

(2) Using EEMD method to decompose the vibration signal into IMFs.

(3) Extract the first $N$ IMFs to form the initial feature matrix $A$, apply SVD technique to $A$ to generate the normalized feature vector $T=\left[p_{1}, p_{2} \cdots p_{N}\right]$, where

$$
p_{i}=\frac{\sigma_{i}^{2}}{p}, p=\sum_{i=1}^{N} \sigma_{i}^{2}
$$

$\sigma_{i}^{2}$ is the singular value of $A$.
(4) For four different fault condition, built three classifiers to conduct three categories. The three categories schematics are shown in Figure 4.

(5) The feature vector is input to SVM to specify the fault type. Output the result.

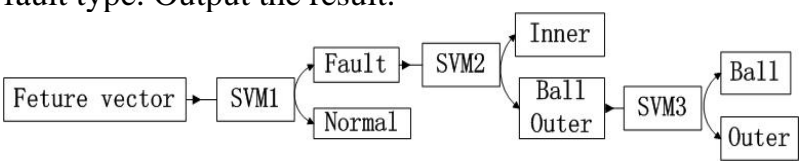

Figure 4. The three categories schematics

\section{2 Experimental results}

Experiment datas are from USA Case Western Reserve University[10]. The bearings (6205-2RSSKF) were seeded with fault by electro-discharge machining. Three single point defects (inner race (IR), ball(B) and outer race(OR)) with diameters 0.007 in. were introduced separately and the data were collected at motor speeds of 1797rpm. The data 97.mat(N),105.mat(IR), 118.mat(B) and 130.mat(OR) are chose in this paper. From each data, 20 samples are intercepted, and the length of each sample is 2048 .

Each sample was decomposed by EEMD (the number of ensemble is 100 and the amplitude of the added white noise is 0.2 ). After analyzing the IMFs, it was found that the first 5 IMFs almost contain all the fault information. So the first 5 IMFs of each sample are chose to generate the feature vector. For each working condition (20 samples), 10 samples were randomly selected as training sample, and the rest 10 samples were selected as testing sample, 8 training samples and 4 testing sample of each

\begin{tabular}{|c|c|c|c|c|c|c|}
\hline \multirow{2}{*}{ Samples } & \multirow{2}{*}{ Fault type } & \multicolumn{5}{|c|}{ Singular value feature vector } \\
\hline & & $p_{1}$ & $p_{2}$ & $p_{3}$ & $p_{4}$ & $p_{5}$ \\
\hline \multirow{8}{*}{$\begin{array}{l}\text { Training } \\
\text { sample }\end{array}$} & \multirow{2}{*}{$\mathrm{N}$} & 0.2987 & 0.1744 & 0.1417 & 0.1006 & 0.0884 \\
\hline & & 0.2992 & 0.1525 & 0.1444 & 0.1204 & 0.0807 \\
\hline & \multirow{2}{*}{ IR } & 0.5205 & 0.2242 & 0.1269 & 0.0438 & 0.0287 \\
\hline & & 0.5261 & 0.2189 & 0.1241 & 0.0486 & 0.0276 \\
\hline & \multirow{2}{*}{ B } & 0.5818 & 0.1278 & 0.1083 & 0.0754 & 0.0484 \\
\hline & & 0.5823 & 0.1295 & 0.1097 & 0.0648 & 0.0465 \\
\hline & \multirow{2}{*}{ OR } & 0.6859 & 0.0929 & 0.0605 & 0.0399 & 0.0314 \\
\hline & & 0.6873 & 0.0875 & 0.0674 & 0.0393 & 0.0319 \\
\hline \multirow{4}{*}{$\begin{array}{l}\text { Testing } \\
\text { sample }\end{array}$} & $\mathrm{N}$ & 0.3090 & 0.1574 & 0.1502 & 0.1036 & 0.0816 \\
\hline & IR & 0.5184 & 0.2242 & 0.1289 & 0.0433 & 0.0287 \\
\hline & B & 0.5945 & 0.1295 & 0.1109 & 0.0569 & 0.0502 \\
\hline & OR & 0.6836 & 0.0849 & 0.0558 & 0.0411 & 0.0388 \\
\hline
\end{tabular}
working condition are shown in Table 2.

Table 2. Training samples and testing samples

The SVM are trained by training sample and the testing sample are used to test the SVM, the test results are shown in Table 3. In order to verify the performance of SVM, BP neural network fault classifier are generated. The results of the two classifier are shown in Table 4. From Table 4 we can find not only testing time but also accuracy that SVM is better than BP network.
Table 3. The test classification results of SVM

\begin{tabular}{|c|c|c|c|c|c|}
\hline $\begin{array}{c}\text { Sample } \\
\text { No. }\end{array}$ & $\begin{array}{c}\text { Fault } \\
\text { type }\end{array}$ & SVM1 & SVM2 & SVM3 & Results \\
\hline $1-10$ & N & +1 & - & - & N \\
\hline $11-20$ & IR & -1 & +1 & - & IR \\
\hline $21-30$ & B & -1 & -1 & +1 & B \\
\hline $31-40$ & OR & -1 & -1 & -1 & OR \\
\hline
\end{tabular}


Table 4. The performance comparison of SVM and BP network

\begin{tabular}{|c|c|c|c|c|c|c|c|}
\hline \multirow{2}{*}{ Classifier } & \multirow{2}{*}{$\begin{array}{c}\text { No. of testing } \\
\text { sample }\end{array}$} & \multirow{2}{*}{$\begin{array}{c}\text { No. of training } \\
\text { sample }\end{array}$} & \multirow{2}{*}{ Time/s } & \multicolumn{4}{|c|}{ Accuracy/\% } \\
\cline { 5 - 8 } & & & & $\mathrm{N}$ & $\mathrm{IR}$ & $\mathrm{B}$ & OR \\
\hline SVM & 10 & 10 & 0.0029 & 100 & 100 & 100 & 100 \\
\hline BP network & 10 & 10 & 1.3412 & 100 & 80 & 80 & 100 \\
\hline
\end{tabular}

\section{Conclusion}

In this paper, a method based on EEMD singular entropy and SVM is presented for diagnosis of bearing. The original signals are adaptively decomposed into a number of IMFs by EEMD. Then the singular value of each IMFs are calculated to generate the feature vector. At last the SVM are used to specify the fault type. The experiment results show that the EEMD method is of great use in processing the non-linear signals, and the proposed method can effectively specify the fault type and it is better than BP neural network.

\section{References}

[1] Yang Yu, Yu Dejie, Cheng Jusheng. Rolling bearing fault diagnosis method based on EMD .J. C J M E. 15,908(2004)

[2] Huang N E, Shen Z, Long S R, et al.The empirical mode decomposition and the Hilbert spectrum for nonlinear and non-stationary time series analysis.J. Proceeding of Royal Society London, Series A. 454,903(1998)

[3] Wu Z, Huang N E. Ensemble empirical mode decomposition:a noise-assisted data analysis method.J. Advances in Adaptive Data Analysis, 1,1(2009)

[4] Zhang Chao, Chen Jianjun, Guo Xun. A gear fault diagnosis method based on EMD energy entropy and SVM.J. Journal of Vibration and Shock, 29,216 (2010)

[5] Cheng Junsheng, Yu Dejie, Yang Y. Fault diagnosis approach based on Intrinsic mode singular value decomposition and support vector machine.J. Acta Automatica Sinica, 32,475 (2006)

[6] Zhang Xiaoyuan, Zhou Jianzhong. Multi-fault diagnosis for rolling element bearings based on ensemble empirical mode decomposition and optimized support vector machines.J. Mechanical Systems and Signal Processing ,41,127 (2013)

[7] Wang Jindun, Chen Lue, Qu Wei. Research on fault week signal feature extraction based on EEMD method.J. Electronic Design Engineering, 20,72 (2012)

[8] Gao Qingqing, Jia Minping. EEMD Method based on singular value spectral entropy in fault diagnosis of roating machine.J. Journal of Southeast University, 41,998(2011)

[9] Deng Naiyang, Tian Yingjie. Support vector machine -theory, algorithm and development(Science Press, Beijing, 2009)

[10]http : // www.eecs.cwru.euc /laboratory/bearing/ welcome_overview. htm 